\title{
Utility of plasma cell-free DNA for de novo detection and quantification of clonal hematopoiesis
}

Fernanda Gutierrez-Rodrigues, Isabel Beerman, ${ }^{2}$ Emma M. Groarke, Bhavisha A. Patel, Nina Spitofsky, ${ }^{1}$ Laura W. Dillon, ${ }^{1}$ Diego Quinones Raffo, ${ }^{1}$ Christopher S. Hourigan, ${ }^{1}$ Sachiko Kajigaya, Luigi Ferrucci ${ }^{2}$ and Neal S. Young ${ }^{1}$

${ }^{1}$ Hematology Branch, National Heart, Lung, and Blood Institute (NHLBI), NIH, Bethesda and ${ }^{2}$ Translational Gerontology Branch, National Institute on Aging, NIH, BRC, Baltimore, MD, USA.

\author{
Correspondence: \\ Fernanda Gutierrez Rodrigues \\ fernanda.rodrigues@nih.gov \\ Received: $\quad$ May 18, 2021. \\ Accepted: $\quad$ August 23, 2021. \\ Prepublished: September 30, 2021. \\ https://doi.org/10.3324/haematol.2021.279230
}

\begin{abstract}
Although cell-free DNA (cfDNA) tests have emerged as a potential non-invasive alternative to bone marrow biopsies for monitoring clonal hematopoiesis in hematologic diseases, whether commercial cfDNA assays can be implemented for the detection and quantification of de novo clonal hematopoiesis in place of blood cells is uncertain. In this study, peripheral plasma cfDNA samples available from patients with aplastic anemia $(n=25)$ or myelodysplastic syndromes $(n=27)$ and a healthy cohort ( $n=107)$ were screened for somatic variants in genes related to hematologic malignancies using a Clinical Laboratory Improvement Amendments-certified panel. Results were further compared to DNA sequencing of matched blood cells. In reported results, $85 \%$ of healthy subjects, $36 \%$ of patients with aplastic anemia and $74 \%$ of patients with myelodysplastic syndromes were found to have somatic cfDNA variants, most frequently in DNMT3A, TET2, ASXL1 and SF3B1. However, concordance between cfDNA and blood cell findings was poor for the detection of clonal hematopoiesis when the allele frequency of the variants was $<10 \%$, which was mostly observed in the healthy and aplastic anemia cohorts but not in patients with myelodysplastic syndromes. After filtering data for potential artifacts due to low variant allele frequency and sequencing depth, the frequency of clonal hematopoiesis in cfDNA from healthy individuals and patients with aplastic anemia decreased to $52 \%$ and $20 \%$, respectively. cfDNA and matched blood cells were not interchangeable for tracking changes in allele burdens as their agreement by Bland-Altman analysis was poor. A commercial cfDNA assay had good performance for de novo detection of clonal hematopoiesis in myelodysplastic syndromes, but showed no advantage over blood cells in diseases with low allele burdens or in healthy individuals.
\end{abstract}

\section{Introduction}

Plasma circulating cell-free DNA (cfDNA) has been used as a source of tumor-derived DNA in peripheral blood (PB) for liquid biopsies., ${ }^{1,2}$ Commercial liquid biopsies based on massively parallel sequencing have been utilized in practice as they are minimally invasive, and tumor-derived cfDNA shows a good correlation with tissue genotypes. ${ }^{3-5}$ However, more than $50 \%$ of somatic variants identified in circulating DNA derive from blood cells and are consistent with clonal hematopoiesis $(\mathrm{CH}),{ }^{4,6}$ a phenomenon in which hematopoietic blood cells acquire variants in genes recurrently mutated in hematologic malignancies. ${ }^{7-10}$

$\mathrm{CH}$ occurs with normal aging, and healthy individuals have $\mathrm{CH}$ of indeterminate potential (CHIP), defined as a clonal population at variant allele frequency $(V A F) \geq 2 \%$ in blood, and mostly associated with a small subset of genes: DNMT3A, TET2, ASXL1, JAK2 and TP53.11 The prevalence of CHIP is agedependent, with the condition being rare until middle age but ubiquitous in individuals older than 60 years when error- correcting sequencing approaches are used for screening.,72 $\mathrm{CH}$ has been used as a predictor for development of blood cancers and associated with an increased risk of cardiovascular disease..$^{710,13-15}$ The particular genes mutated, the number of variants and clone sizes influence the likelihood of healthy individuals with CHIP progressing to develop a hematologic malignancy. ${ }^{10,14-16}$

In hematologic diseases, $\mathrm{CH}$ has been used as a predictor of clinical outcomes and for risk stratification of patients. ${ }^{17-19}$ In aplastic anemia (AA), pathogenic variants in PIGA and BCOR correlate with a better response to immunosuppressive therapy whereas patients with variants in RUNX1, TP53, DNMT3A and $A S X L 1$ have worse outcomes; typically the median VAF of these variants is $9 \% .^{19}$ Genomic data are also incorporated into prognostic models for myelodysplastic syndromes (MDS); variants are commonly found at higher VAF (>30\%) and in genes such as ASXL1, DNMT3A, NRAS, RUNX1, U2AF1 and TP53, which are associated with poor prognosis. ${ }^{17-20}$

In this context, the use of plasma cfDNA sequencing assays for the detection and quantification of $\mathrm{CH}$ has emerged as a 
potential non-invasive alternative to bone marrow (BM) biopsies in MDS and AA. In addition, plasma is underused in many laboratories and may be a source of DNA for many retrospective $\mathrm{CH}$ studies in asymptomatic individuals when blood samples are not available. A few studies have shown that both PB and BM cells can be used for detection and quantification of $\mathrm{CH}^{10}$ however, it is not yet known whether cfDNA is equally accurate. Previous experiences with liquid biopsies have demonstrated that highly sensitive assays are required to reliably detect small clonal populations, and poor congruence between Clinical Laboratory Improvement Amendments (CLIA)-certified commercial cfDNA assays has often been reported. ${ }^{21-23}$ These findings imply that detection of de novo $\mathrm{CH}$ in cfDNA may be troublesome, as CHIP variants can often be seen at low frequency and no matched samples or previous genotyping data would be available for validating cfDNA results.

To investigate the cfDNA performance of a commercial CLIAapproved targeted sequencing panel of genes related to $\mathrm{CH}$ and hematologic malignancy in detecting and quantifying de novo $\mathrm{CH}$ variants, we screened healthy individuals from the Baltimore Longitudinal Study of Aging (BLSA) and patients with AA and MDS. Reported cfDNA results were validated against DNA-sequencing from matched blood cells.

\section{Methods}

\section{Cohorts and samples}

EDTA-PB plasma for cfDNA assay was available for 107 healthy individuals, including 96 participants of BLSA at the National Institute of Aging (NIA, NCT00233272) and 11 healthy volunteers at the National Heart, Lung, and Blood Institute (NHLBI). EDTA-PB samples were also collected at diagnosis from patients with AA $(n=25)$ or MDS $(n=27)$ from the Hematology Branch marrow failure clinic at the NHLBI (NCT00932156 and NCT01623167). Matched PB or BM cells collected at the same time as peripheral plasma samples were used for comparative analyses. In the healthy cohort, only 25 individuals had matched samples from PB mononuclear cells (PBMC, $n=15)$ or granulocytes $(n=10)$. In the AA and MDS cohorts, paired $B M$ cells from diagnosis and serial time-points were used as matched samples (AA, $n=53$ and MDS, n=55) (Online Supplementary Figure S1).

This study was approved by the Institutional Review Boards of the NHLBI and NIA. All samples were collected according to the Declaration of Helsinki and written consent was obtained from all participants or their legal guardians.

\section{Commercial massively parallel sequencing of targeted genes}

cfDNA and PB/BM samples were screened for somatic variants in hematologic neoplasm-related genes using the GTCHematology profile (Genomic Testing Cooperative, CA, USA), a commercial CLIA panel that includes genes commonly associated with CHIP (CHIP genes) and cancer and hematologic neoplasia (non-CHIP genes) (Online Supplementary Table S1). ${ }^{16,24}$

The somatic origin of variants at VAF $>35 \%$ reported by the commercial assay was confirmed by targeted DNA sequencing of sorted $\mathrm{CD}^{+}$cells or PBMC/granulocytes; variants present in these fractions at similar VAF to CfDNA were considered germline (Online Supplementary Tables S2-S4). Of note, the cfDNA GTC-Hematology profile assay was CLIA-validated for detecting abnormalities in MDS and AA but not for non-myeloid neoplasms; therefore, samples from healthy individuals were screened for research purposes only.

\section{Comparative analysis of cell-free DNA and matched peripheral blood or bone marrow samples}

Sensitivity, specificity and positive predictive value of the CLIA-certified cfDNA test were assessed in comparison to matched PB or BM samples. Likely germline variants were also included in this comparative analysis. Concordance among matched samples was evaluated at a variant level with each variant classified as true positive, false positive, false negative and true negative (Figure $2 A$ ). The performance of the cfDNA assay in quantifying both somatic and likely germline variants was assessed by linear regression and the Bland-Altman analysis, a more accurate method to evaluate the agreement between paired values. ${ }^{25}$ Statistical analysis was performed using GraphPad Prism v5 (GraphPad Software Inc, CA, USA).

\section{Stringent criteria for filtering likely false positive and false negative variants}

Ultra-deep error-correcting sequencing was performed in our institution as previously described to validate the CLIA results of selected samples from healthy individuals for whom we had available materials. ${ }^{26}$ Results obtained from genetic reports were refined by stringent criteria to filter variants that were below the VAF and read depth thresholds of confirmed negative variants by the ultra-deep error-correcting sequencing.

Detailed experimental procedures and analytical methods are described in the Online Supplementary Data.

\section{Results}

\section{Clonal landscapes of cell-free DNA variants in healthy individuals, and patients with aplastic anemia or myelodysplastic syndromes}

Among 107 healthy subjects (median age 72 years; range, 24-96) screened for somatic variants in cfDNA, 91 (85\%) had at least one variant. The majority $(75 / 107$ subjects, $71 \%)$ had one $(n=27)$ or two or more $(n=48)$ variants in CHIP genes, most frequently in DNMT3A, TET2, TP53 and 
ASXL1 (Figure 1A, Online Supplementary Figure S2). However, $50 / 107$ subjects (47\%) also had somatic variants in non-CHIP genes, and 16 of them (16/50) had variants in non-CHIP genes only (Online Supplementary Table S2). The frequency of somatic cfDNA variants in CHIP genes, DTA genes (DNMT3A, TET2 and ASXL1) and non-CHIP genes increased with age and $\mathrm{CH}$ was observed in more than $60 \%$ of individuals older than 40 years (Figure 1B).

Among the 25 patients with AA (median age 51 years; range, 13-82), nine (36\%) were found to have somatic cfDNA variants. Most of them had one $(n=1)$ or two $(n=5)$ cfDNA variants in CHIP genes (6/25, 24\%), most commonly in BCOR and SF3B1 (Figure 1C). Among 27 MDS patients (median age 63 years; range, 35-85), 20 (74\%) had somatic cfDNA variants, including 16/27 (60\%) with variants in CHIP genes. Patients had one $(n=10)$ or two or more $(n=6)$ cfDNA variants, TET2, SF3B1 and $A S X L 1$ being most frequent (Figure 1D). Also, 5/32 AA and 9/27 MDS patients were found to have cfDNA variants in non-CHIP genes (Figure 1C, D). Four MDS and three AA patients had cfDNA variants exclusively in non-CHIP genes (Online Supplementary Tables S3 and S4).

\section{Concordance between cell-free DNA and blood cells was limited by the allele frequency of the variants} In paired samples from healthy individuals $(n=25)$, most cfDNA variants identified were not found in matched cells (i.e, they were false-positives), and true positives were likely germline variants (median VAF $47 \%$ in all cfDNA, PBMC and granulocytes) (Figure 2B). Sensitivity was defined as a proportion of variants that was correctly detected in cfDNA, and specificity was defined as the probability that a given patient was correctly reported with no variants. The positive predictive value was defined as the probability of a variant detected in cfDNA being also detected in blood cells. In this cohort, sensitivity, specificity and positive predictive value of the cfDNA assay were low (32\%, 26\% and 15\%, respectively), and most discordant variants had median VAF $<10 \%$ (Table 1, Figure 2B). Here, the median VAF of variants identified in the healthy cohort was $2.5 \% \pm 10 \%$ (95\% confidence interval [Cl]: 2-4] and both the sensitivity and positive predictive value of the assay for detecting variants at VAF $<10 \%$ were poor (Figure $2 \mathrm{E}$, Table 1 ).

In contrast to healthy subjects, most cfDNA variants found in AA and MDS patients were true positives with high concordance between paired samples. Sensitivity, specificity and the positive predictive value of cfDNA in comparison to $\mathrm{BM}$ cells were greater than $70 \%$ and $85 \%$ in AA and MDS patients, respectively (Table 1). Most of the discordant pairs from AA- and MDS-matched samples were at lower VAF (Figure 2B). In all cohorts, the median VAF of discordant pairs was significantly lower than the VAF of true positives (7.6\% [95\% Cl: 4-10] vs. 33\% [95\% Cl:
28-37]; t-test, $P<0.001)$, and most discordant variants were at VAF $<10 \%$ (upper CI of VAF from discordant pairs). Indeed, discordance observed in paired samples correlated with a proportion of discordant variants at VAF $<10 \%$ in each cohort, regardless of disease types (Table 1). Sensitivity, specificity and positive predictive value were very high in MDS, in which most variants were seen at higher VAF (median VAF=32\%, 95\% Cl: 27-42) (Figure $2 \mathrm{E})$. In contrast, the lowest concordance was seen in healthy subjects, in whom most discordant variants were seen at VAF $<10 \%$ (Figure $2 \mathrm{~B}$ ).

\section{Analytical factors associated with cell-free DNA screening were related to assay discordance}

We next investigated whether discordance also correlated with sequencing depth, an analytical factor that can lead to high rates of sequencing artifacts. In all cohorts, read depth of deduplicated reads in true positives was significantly higher (482X, 95\% Cl: 368X-578X) (Figure 2D) than in false positives and false negatives (110X, 95\% Cl: 89X$143 X)$. To confirm these findings, we validated variants found in $\mathrm{PB}$ cells from two healthy subjects using an ultra-deep error-correcting sequencing panel with 13 genes: plasma samples were not available for this inhouse validation. Four out of six variants initially identified in these PB cells at a median VAF of $2.5 \%$ (range, $2-3.6 \%$ ) and a median read depth of 246X (range, 104X-347X) were all confirmed negative (Figure 2D). In this validation, 95\% of confirmed negative variants were at VAF $<3.5 \%$ and were sequenced at coverages below <350X, which may represent a limit of detection of this commercial assay (Figure 2D). Of note, as stated by the vendor, the assay has a typical sensitivity of $5 \%$ for overall variant detection and $3 \%$ for detecting specific somatic variants in "hotspots." However, somatic variants with an allele frequency as low as $0.9 \%$ were reported by the laboratory, and included in our concordance analysis.

We found no specific genes or types of variants associated with assay discordance. When combining all three cohorts, false positive and false negative cfDNA variants were found mostly in ASXL1, TET2, SF3B1 and DNMT3A, and included missense, stop-gain and frameshift variants. The remaining discordant variants were seen in a broad spectrum of genes (Online Supplementary Figure S3).

\section{Stringent criteria to filter out discordant variants improved cell-free DNA concordance with blood cells}

To improve concordance between paired samples, we further refined our analysis by filtering out variants that were below the VAF and read depth thresholds of confirmed negative variants by error-correcting sequencing (likely artifacts from the cfDNA CLIA assay). We first discarded variants at VAF $<1 \%$ and read depth $<700 \mathrm{X}$, and variants at VAF $<3.5 \%$ and read depth $<400 X$ (more strin- 
A

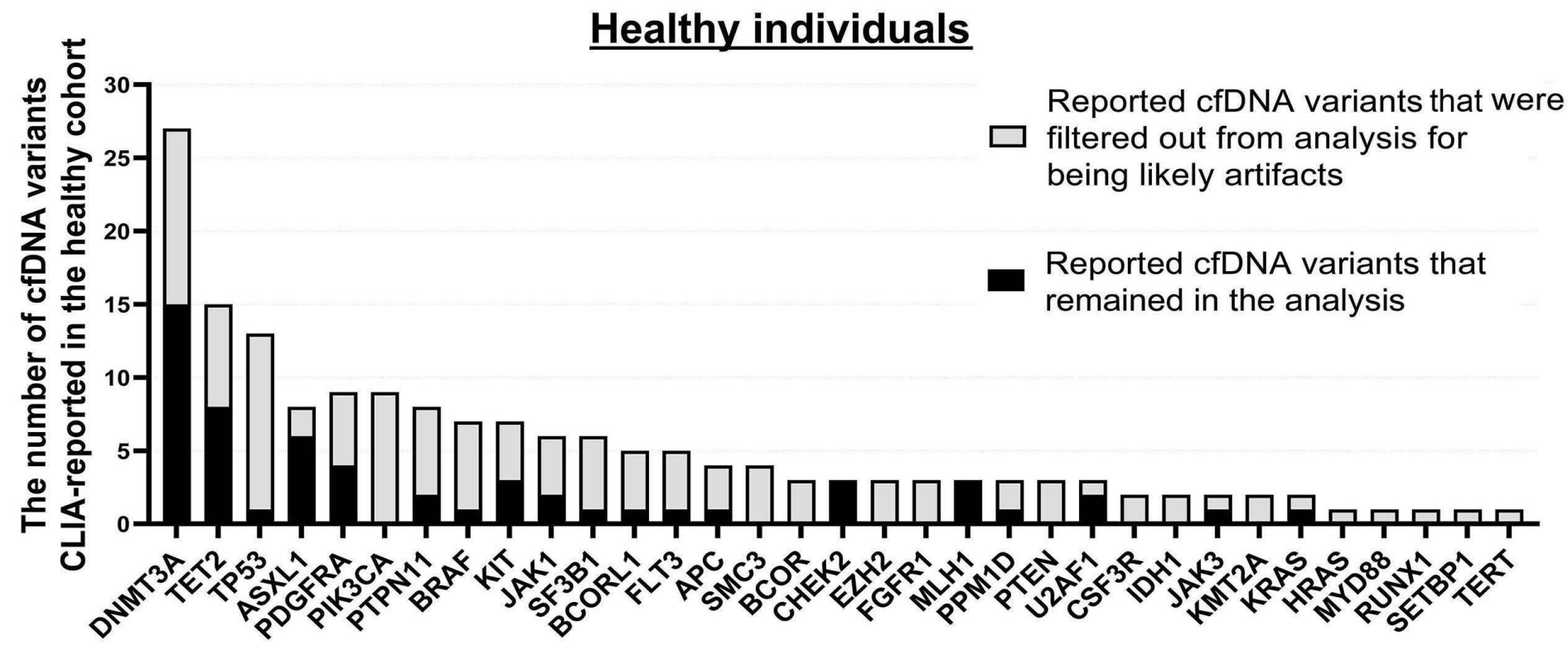

B

Original data from genetic reports

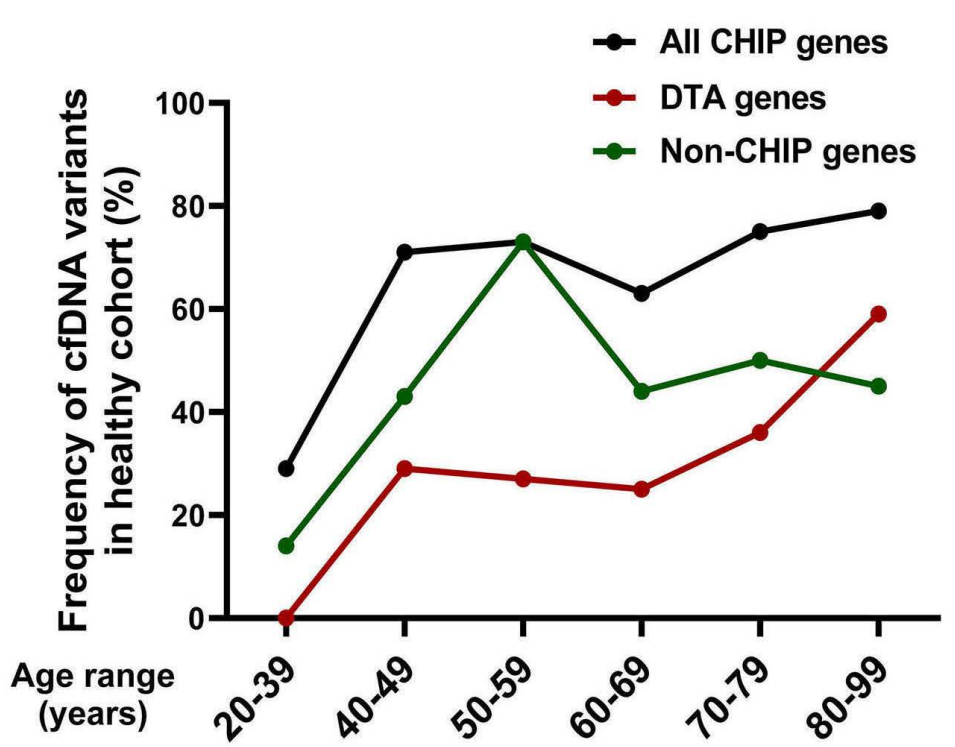

$\begin{array}{cllllll}\text { No. } & 7 & 7 & 11 & 16 & 36 & 29\end{array}$

C

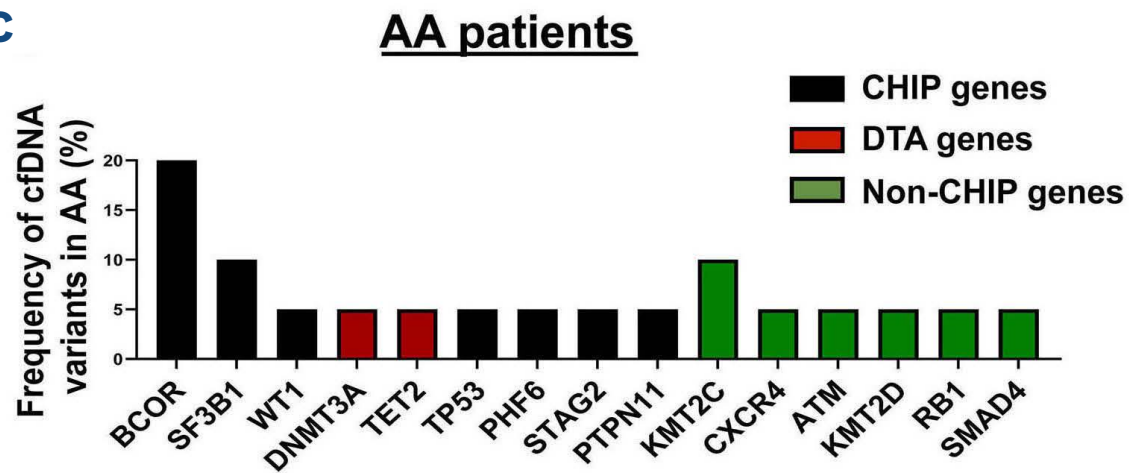

D

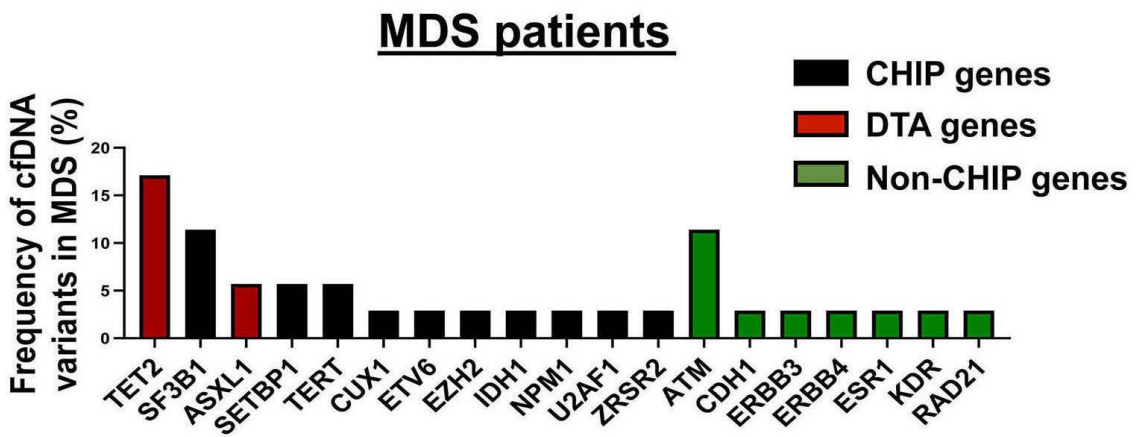

$\mathbf{E}$

\section{Filtered data}

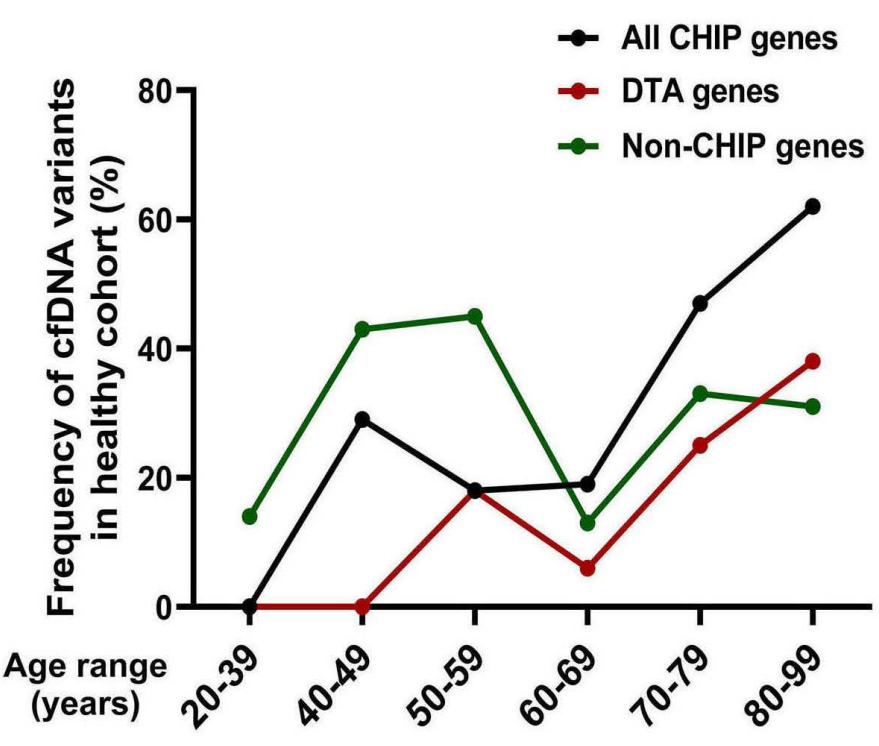

$\begin{array}{ccccccc}\text { No. } & 7 & 7 & 11 & 16 & 36 & 29\end{array}$

$\mathbf{F}$

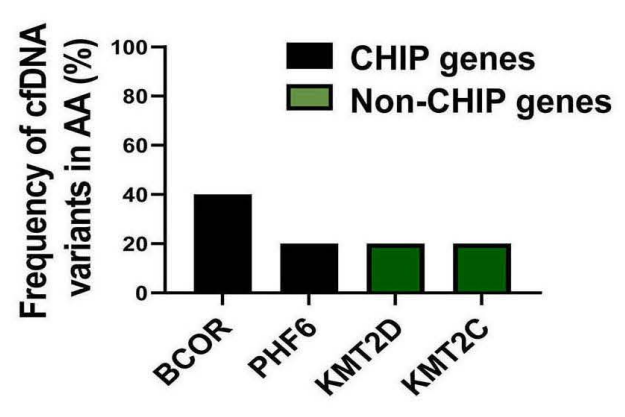


Figure 1. Clonal landscapes of somatic cell-free DNA variants detected in healthy individuals and patients with aplastic anemia or myelodysplastic syndromes. (A) The most frequently mutated genes in 107 healthy subjects obtained from genetic reports. Cell-free (cfDNA) variants were mostly found in DNMT3A, TET2, TP53 and ASXL1 (full lists of genes are shown in Online Supplementary Figure S2). However, during analysis, the original data from genetic reports were filtered out to remove variants that were likely false positive and false negative, due to low variant allele frequency (VAF) and low sequencing coverage. Variants originally reported by the Clinical Laboratory Improvement Amendments (CLIA) assay were filtered out from analysis if present at $\mathrm{VAF}<10 \%$ and had been sequenced at a read depth $<100 X$, or were present at VAF $<3.5 \%$ and had been sequenced at a read depth <400X. After data filtering, cfDNA variants were mostly found in DNMT3A, TET2, ASXL1 (DTA genes) and KIT. (B) Frequency of cfDNA variants by age ranges. Frequency of variants in genes commonly associated with clonal hematopoiesis of indeterminate potential (CHIP), including DTA genes, and non-CHIP genes. Approximately $60 \%$ of individuals aged 40 years or older were found to have clonal hematopoiesis in cfDNA. (C) The most frequently mutated genes in 25 patients with aplastic anemia at enrollment in the NCT00932156 trial or at diagnosis. (D) The most frequently mutated genes in 27 patients with myelodysplastic syndromes at enrollment in the NCT01623167 trial. (E) Frequency of cfDNA variants by age ranges after original data were filtered to remove variants that were likely false positive and false negative, due to low VAF and low sequencing coverage. Frequency of variants in both CHIP genes, including DTA genes alone but also in non-CHIP genes, increased with aging. Approximately $30 \%$ of individuals aged 40 years or older were found to have clonal hematopoiesis in cfDNA. (F) The most frequently mutated genes in 25 patients with aplastic anemia after data filtering. BM: bone marrow; PB: peripheral blood; AA: aplastic anemia; MDS: myelodysplastic syndromes.

Table 1. Concordance between cell-free DNA and bone marrow or peripheral blood cells for detection of clonal hematopoiesis.

\begin{tabular}{|l|c|c|c|c|c|c|c|}
\hline TP & FN & FP & TN & $\begin{array}{c}\text { Sensitivity Specificity } \\
\text { PPV }\end{array}$ & $\begin{array}{c}\text { Discordant } \\
\text { variants } \%\end{array}$ \\
\hline
\end{tabular}

\section{Data originally reported by the CLIA- certified assay}

\begin{tabular}{|c|c|c|c|c|c|c|c|c|}
\hline $\begin{array}{l}\text { Healthy individuals } \\
\text { VAF }>10 \% \\
\text { VAF }<10 \%\end{array}$ & $\begin{array}{l}7 \\
6 \\
1\end{array}$ & $\begin{array}{c}16 \\
1 \\
15\end{array}$ & $\begin{array}{l}41 \\
26 \\
15\end{array}$ & 4 & $\begin{array}{c}30 \\
86 \\
6\end{array}$ & 9 & $\begin{array}{c}15 \\
19 \\
6\end{array}$ & $\begin{array}{l}89.1 \\
42.2 \\
46.9\end{array}$ \\
\hline $\begin{array}{l}\text { Aplastic anemia } \\
\qquad \begin{array}{l}\text { VAF }>10 \% \\
\text { VAF }<10 \%\end{array}\end{array}$ & $\begin{array}{l}25 \\
11 \\
14\end{array}$ & $\begin{array}{l}6 \\
0 \\
6\end{array}$ & $\begin{array}{c}10 \\
0 \\
10\end{array}$ & 33 & $\begin{array}{c}81 \\
100 \\
70\end{array}$ & 77 & $\begin{array}{c}71 \\
100 \\
58\end{array}$ & $\begin{array}{c}39 \\
0 \\
39\end{array}$ \\
\hline $\begin{array}{l}\text { Myelodysplastic syndromes } \\
\text { VAF }>10 \% \\
\text { VAF }<10 \%\end{array}$ & $\begin{array}{c}85 \\
80 \\
5\end{array}$ & $\begin{array}{l}15 \\
7 \\
8\end{array}$ & $\begin{array}{l}0 \\
0 \\
0\end{array}$ & 11 & $\begin{array}{l}85 \\
92 \\
38\end{array}$ & 100 & $\begin{array}{l}100 \\
100 \\
100\end{array}$ & $\begin{array}{l}15 \\
7 \\
8\end{array}$ \\
\hline \multicolumn{9}{|c|}{ Concordance after data filtering ${ }^{*}$} \\
\hline $\begin{array}{l}\text { Healthy individuals } \\
\text { VAF }>10 \% \\
\text { VAF }<10 \%\end{array}$ & $\begin{array}{l}7 \\
6 \\
1\end{array}$ & $\begin{array}{l}2 \\
1 \\
1\end{array}$ & $\begin{array}{c}34 \\
25 \\
9\end{array}$ & 9 & $\begin{array}{l}78 \\
86 \\
50\end{array}$ & 21 & $\begin{array}{l}17 \\
19 \\
10\end{array}$ & $\begin{array}{l}83.7 \\
60.5 \\
23.3\end{array}$ \\
\hline $\begin{array}{l}\text { Aplastic anemia } \\
\text { VAF>10\% } \\
\text { VAF<10\% }\end{array}$ & $\begin{array}{l}17 \\
11 \\
6\end{array}$ & $\begin{array}{l}3 \\
0 \\
3\end{array}$ & $\begin{array}{l}1 \\
0 \\
1\end{array}$ & 39 & $\begin{array}{c}85 \\
100 \\
67\end{array}$ & 98 & $\begin{array}{c}94 \\
100 \\
86\end{array}$ & $\begin{array}{c}19 \\
0 \\
19\end{array}$ \\
\hline $\begin{array}{l}\text { Myelodysplastic syndromes } \\
\text { VAF }>10 \% \\
\text { VAF }<10 \%\end{array}$ & $\begin{array}{c}83 \\
80 \\
3\end{array}$ & $\begin{array}{c}13 \\
11 \\
2\end{array}$ & $\begin{array}{l}1 \\
1 \\
0\end{array}$ & 12 & $\begin{array}{l}86 \\
88 \\
60\end{array}$ & 92 & $\begin{array}{c}99 \\
99 \\
100\end{array}$ & $\begin{array}{c}14.4 \\
12.4 \\
2.1\end{array}$ \\
\hline
\end{tabular}

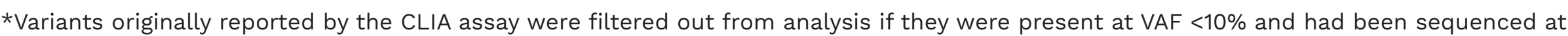

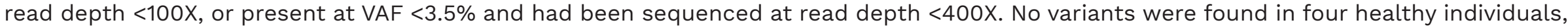

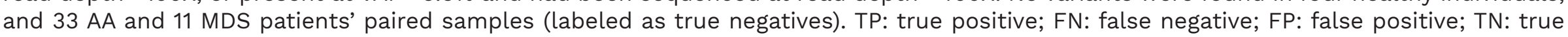
negatives; PPV: positive predictive value; CLIA: Clinical Laboratory Improvement Amendments; VAF: variant allele frequency.

gent than the minimum of $350 \mathrm{x}$ observed as the upper $95 \% \mathrm{Cl}$ for confirmed negative variants). cfDNA variants at a VAF $<10 \%$ were only included in the analysis if the read depth was $>100 X$ (Figure 2D). Of note, the read depth of a variant at the lowest VAF (0.96\%) found in both cfDNA and blood cells was 750X. Overall concordance between cfDNA and matched samples was improved for both healthy individuals and AA patients, but there were no significant changes in MDS paired samples (Table 1).
In the healthy cohort, once filtering criteria were applied, the frequency of subjects with somatic cfDNA variants was lower than that in the original analysis (56/107; 52\% vs. 92/107; 86\%); the frequency of CHIP variants in individuals $>40$ years old also decreased (30\% vs. 60\%) (Figure $1 \mathrm{~B}, \mathrm{E})$. In addition, the frequency of cfDNA variants in non-CHIP genes was no longer related to increased age, and the median allele frequency of the variants increased substantially to $12 \%$ (95\% Cl: 10-15) (Figures $1 \mathrm{E}$ and $2 \mathrm{E}, \mathrm{F}$ ). 
A

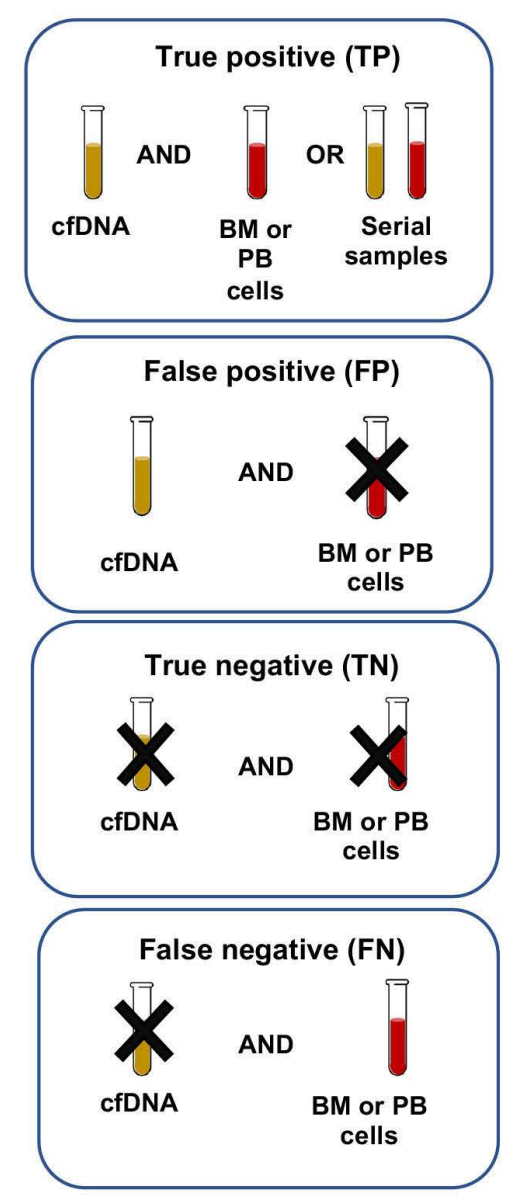

D

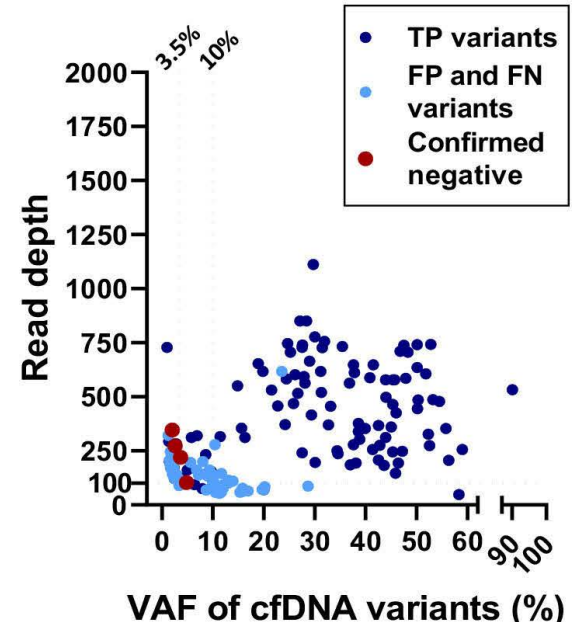

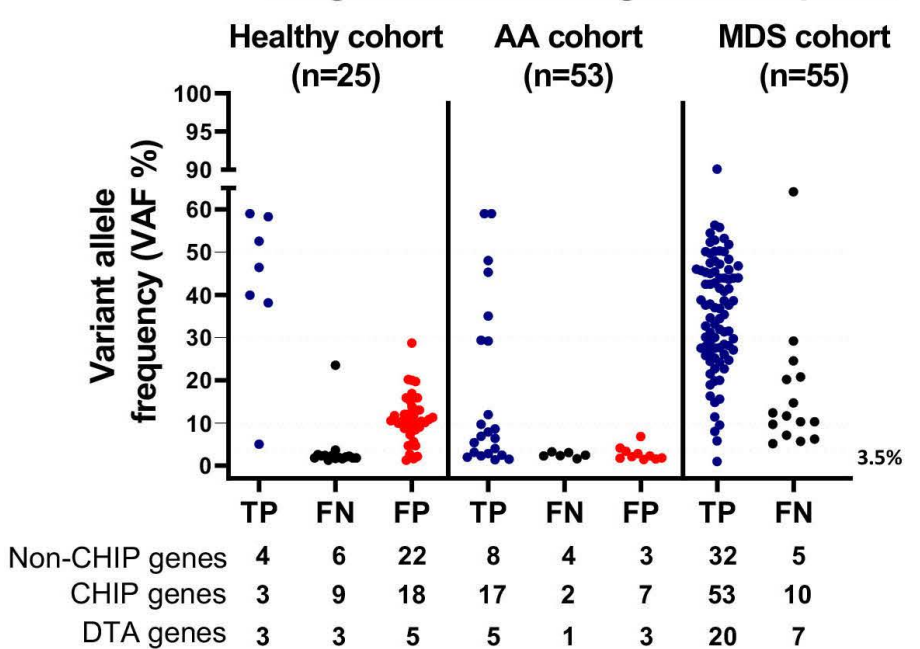

Filtered data

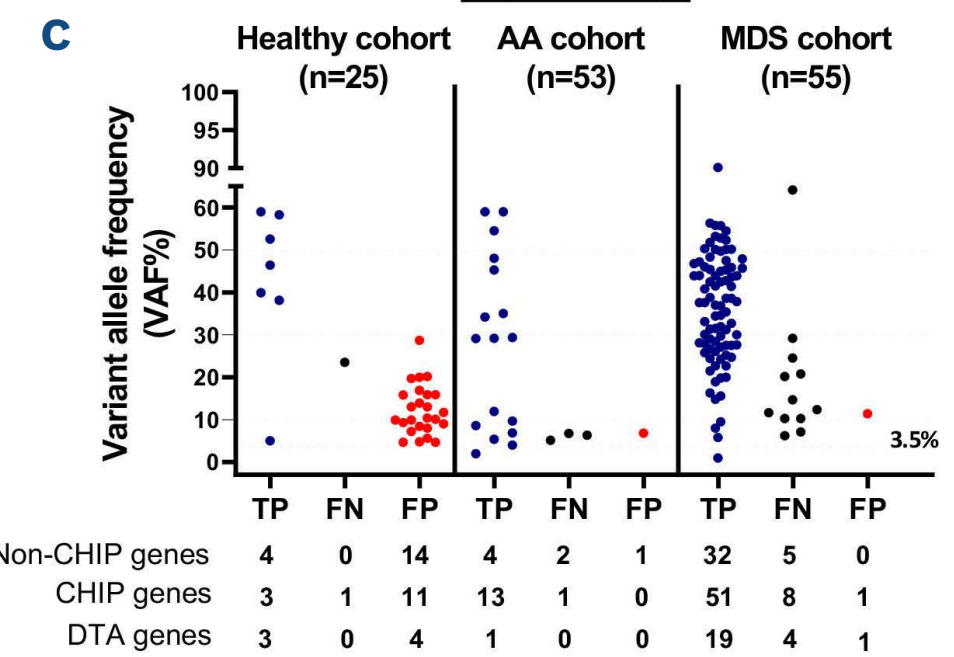

E

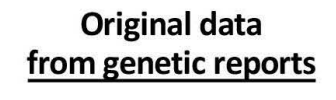

Filtered data
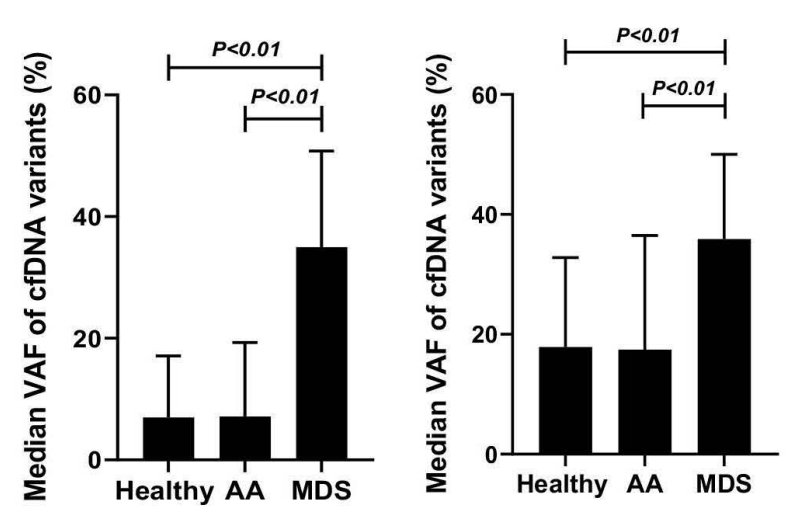

Figure 2. Concordance analysis of variants detected in cell-free DNA and in matched peripheral blood or bone marrow cells. (A) Schematic criteria used to classify cell-free DNA (cfDNA) variants as (i) true positive (TP), if detected in both cfDNA and a matched sample, or a matched sample at a different time-point; (ii) false positive (FP), if detected only in cfDNA; (iii) false negative (FN), if absent in cfDNA but present in the matched-sample; and (iv) true negative (TN), if no variants were found in either cfDNA or the paired samples. (B) Analysis with original data from genetic reports. The figure shows the variant allele frequency (VAF) of TP, FN and FP variants detected in paired samples from healthy individuals $(n=25)$, patients with aplastic anemia (AA, $n=53)$ and patients with myelodysplastic syndromes (MDS, $n=55$ ). VAF of FN variants corresponded to VAF found in peripheral blood (PB) or bone marrow (BM) cells. (C) VAF of TP, FP and FN detected in paired samples that remained in the analysis after original Clinical Laboratory Improvement Amendments (CLIA) data were filtered out to remove variants that were likely FP and FN, due to low VAF and low sequencing coverage. (D) VAF and coverage (read depth) of discordant variants (FP and FN) in comparison to TP variants. Single variants found in both cfDNA and matched blood cells are represented by dark blue circles (TP). Single discordant variants in cfDNA and matched blood cells are indicated with light blue circles (FP and FN). Variants identified in PB cells from healthy individuals and confirmed negative by ultra-deep error-correcting sequencing are represented by red circles. VAF of $10 \%$ and minimal read depth 100X were thresholds that delimited the $95 \%$ confidence interval $(95 \% \mathrm{Cl})$ observed for discordant variants. VAF below $3.5 \%$ and read depth below 400X were the minimum thresholds of the assay sensitivity based on the VAF and read depth of confirmed negative variants (limits of detection of the assay). VAF and read depth of FN variants represent VAF and coverage of variants detected in PB or BM cells. (E) Median VAF with $95 \% \mathrm{Cl}$ of cfDNA variants reported in the CLIA cfDNA assay. Median VAF of cfDNA in healthy controls, and individuals with AA or MDS were $2.5 \%$ (2\%-4\%), $2.6 \%(2 \%-4 \%)$ and $32 \%(27 \%-42 \%)$, respectively. (F) Median VAF with $95 \%$ CI of cfDNA variants that remained in the analysis after original CLIA data were filtered out to remove variants that were likely FP and FN, due to low VAF and low sequencing coverage. Median VAF of cfDNA in healthy controls, and individuals with AA or MDS were $12 \%$ (10\%-15\%), $6.7 \%(2 \%-45 \%)$ and $37 \%(31 \%-42 \%)$, respectively. 
A

\section{Healthy cohort}
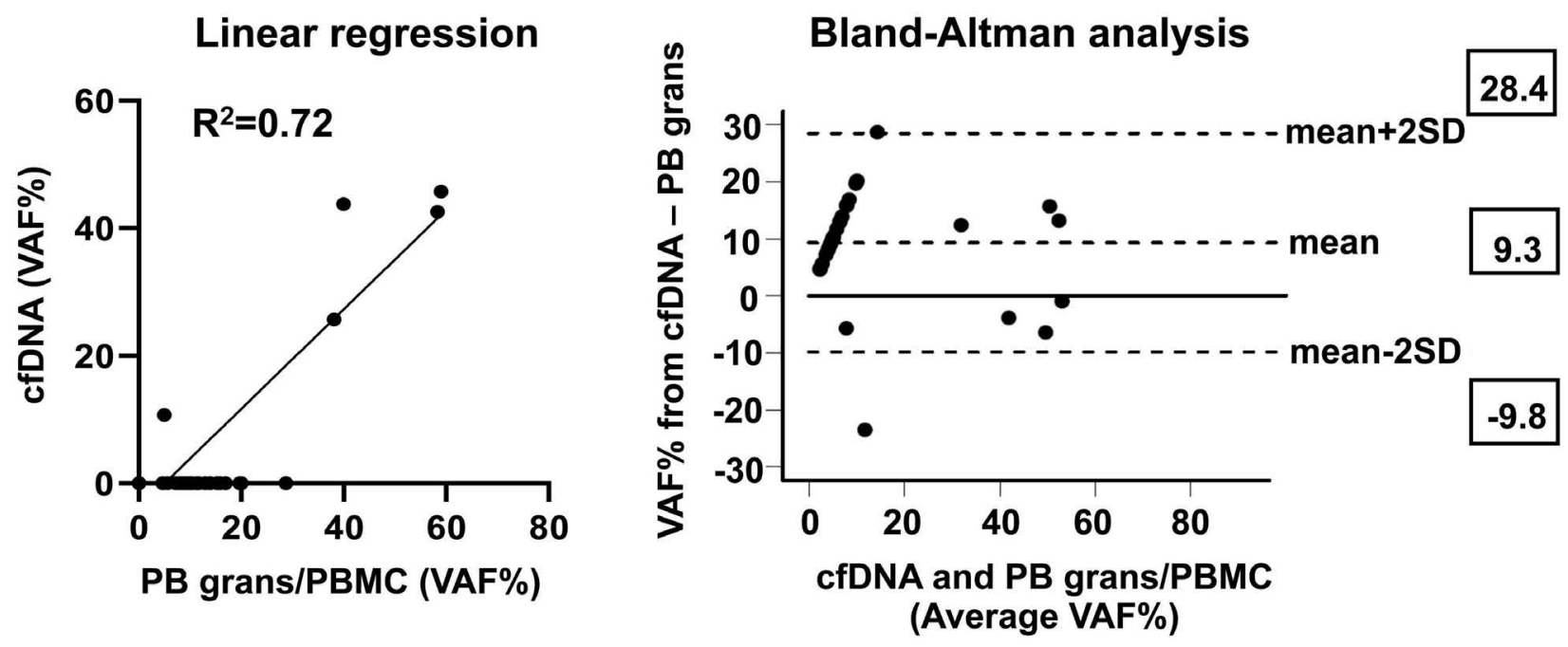

AA cohort

B

Linear regression

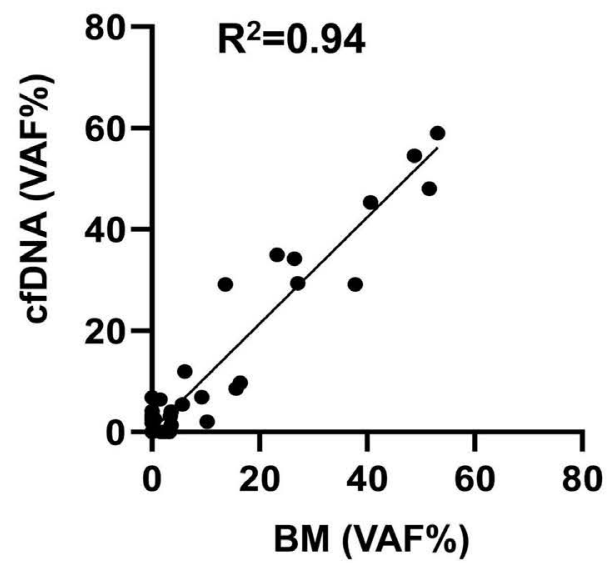

Bland-Altman analysis

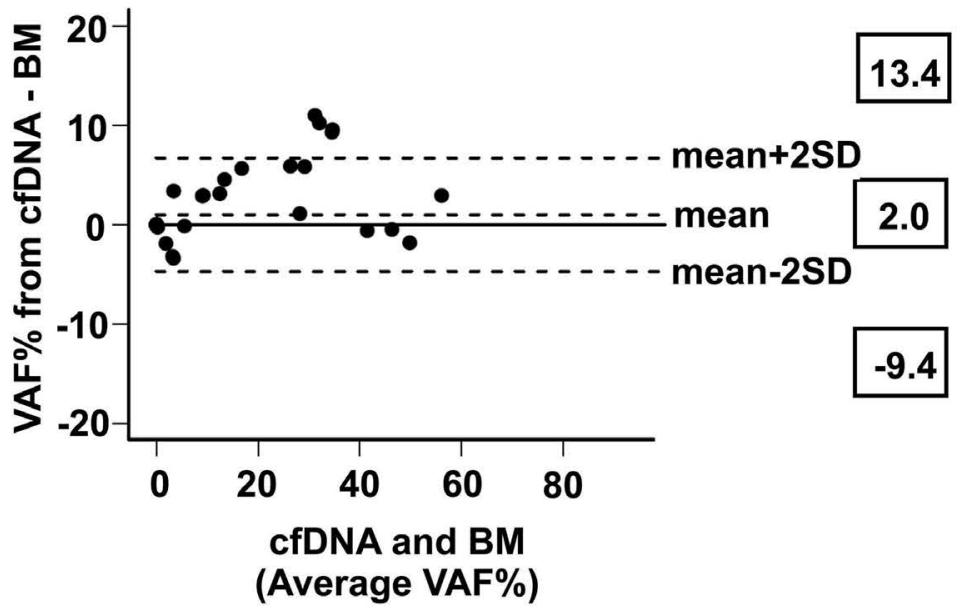

MDS cohort

C

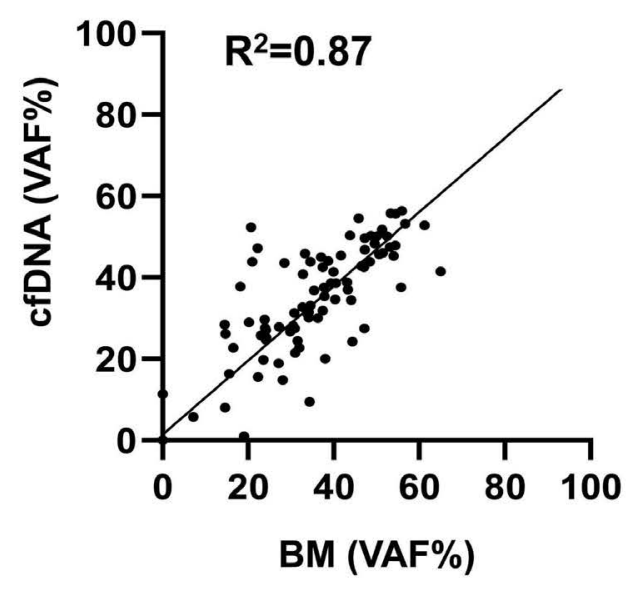

Bland-Altman analysis

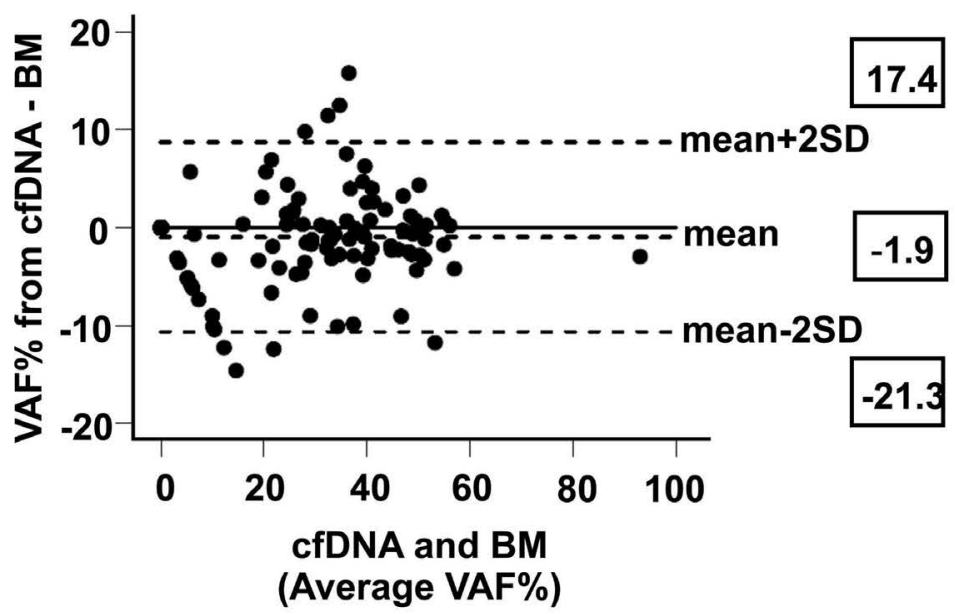

Figure 3. Performance of cell-free DNA for quantification of variants in comparison to matched peripheral blood or bone marrow cells. Linear regression was used to assess a correlation between quantification of clones in paired samples. Agreement analysis by the Bland-Altman method was performed to assess how accurate and precise the quantification of variants in cellfree DNA (cfDNA) were in comparison to matched blood cells. This method calculates the bias, the mean difference between the variant allele frequency (VAF) of matched samples and its standard deviation (SD) to more accurately evaluate agreement between paired values. Bias and SD were later used to define limits of agreement (LoA; mean \pm 2 SD) which represent the 95\% confidence interval of mean differences. Both analyses were performed with paired variants from healthy subjects (A), patients with aplastic anemia (B) and patients with myelodysplastic syndromes (C) who remained in the analysis after data filtering. For Bland-Altman analysis, differences of VAF of individual paired variants detected in cfDNA and peripheral blood or bone marrow cells are plotted against their average VAF (right panels). Mean differences (bias) of VAF and LoA (mean \pm 2 SD) are shown in the graphic. The closer the mean was to zero and the narrower the LoA was, the better agreement between VAF of variants detected in paired samples. PB: peripheral blood; grans: granulocytes; PBMC: peripheral blood mononuclear cells; BM: bone marrow; AA: aplastic anemia; MDS: myelodysplastic syndromes. 
The frequency of $\mathrm{CH}$ in AA also decreased after data filtering $(9 / 25 ; 36 \%$ vs. $5 / 25 ; 25 \%)$. Although the median allele frequency of the variants and the most commonly mutated genes changed slightly in AA after the filtering process (6.7\%, 95\% Cl: $2 \%-45 \%$ ) (Figures $1 \mathrm{~F}$ and $2 \mathrm{E}, \mathrm{F}$ ), no changes were seen in MDS as most variants had high VAF and sequencing coverage (Figure $2 \mathrm{~B}, \mathrm{C}$ ). In MDS, a variant previously found in matched samples and classified as a true positive was reclassified as a false positive after data filtering; in the BM, this variant was of low quality and systematically filtered with stringent criteria (Figure 2C).

\section{Quantification of clonal hematopoiesis in cell-free DNA was not comparable to that in blood cells}

We further investigated whether the VAF of filtered cfDNA variants accurately replicated the VAF in blood cells. Linear regression showed a high correlation between paired samples in all cohorts $\left(\mathrm{R}^{2}>0.7\right)$ (Figure 3$)$. However, agreement between the quantification of $\mathrm{CH}$ in cfDNA and matched blood samples by the Bland-Altman method was poor for all the cohorts (Figure $3 A-C$ ). An average difference (bias) and standard deviation (SD) of a clone size (VAF\%) found in paired samples was $9.3 \% \pm 9.5 \%$ in healthy individuals, $2.0 \% \pm 5.7 \%$ in $\mathrm{AA}$ and $-1.9 \% \pm 6.5 \%$ in MDS. These large variations in the bias and SD translated to a wide range of limits of agreement (mean $\pm 2 \mathrm{SD}$ ); the largest variations were seen in healthy individuals and MDS samples as cfDNA variants over- and under-estimated clone sizes by up to $28 \%$ and $21 \%$, respectively (Figure $3 A$, C). Also, in all cohorts, the bias of some paired values was not within the limits of agreement, suggesting that variations larger than the confidence intervals may often be observed in cfDNA, regardless of disease type.

\section{Healthy individuals with cell-free DNA variants in prognostically adverse genes}

We next assessed whether the presence of variants in genes highly related to an increased risk of developing myeloid malignancies (TP53, RUNX1, IDH1, IDH2 and U2AF1) was associated with clinical outcomes in healthy individuals followed in the BLSA study. Nineteen individuals were initially found to have variants in prognostically adverse genes, but most of these variants had no clinical significance as they were later filtered out due to their low VAF and poor sequencing coverage (especially TP53 and DNMT3A variants) (Figure 4A). After data filtering, only two of ten individuals found with variants in prognostically adverse genes developed hematologic diseases (Figure 4B). HC-26 and HC-64 were found with pathogenic variants in NRAS, U2AF1 and TP53 at VAF $>30 \%$ which were associated with longstanding cytopenias. HC-26 died 2 years following the data collection, reportedly from acute leukemia. Although $\mathrm{HC}-04$ and $\mathrm{HC}-104$ were also found to have pathogenic variants in U2AF1 and IDH1 at VAF around
$15 \%$, the former had a diagnosis of prostate cancer with no evidence of hematologic disease at the time his sample was collected, and this variant may have been present in the bloodstream due to his solid organ malignancy. Similarly, HC-64 had a high VAF TP53 variant, for which a somatic status was not confirmed. This individual had no history of hematologic disease or a solid malignancy but had cytopenias of unknown significance for 9 years before his last follow-up. The remaining cfDNA variants were at very small allele fractions (median VAF 1.2\% with the subjects' median age of 65 years) and none of the individuals was known to have myeloid malignancy at last follow-up, suggesting that the clinical utility of the cfDNA assays for CHIP surveillance in healthy individuals was limited.

\section{Discussion}

The questionable analytical validity of cfDNA assays has raised concerns regarding their clinical utility. Two major issues facing routine implementation of cfDNA tests are the high frequency of discordant variants in matched samples and the lack of characterization of the cfDNA genomic landscapes in both healthy individuals and in specific disease states. ${ }^{2}$ As most studies in this field have focused on precision oncology and detection of tumorderived DNA, the clinical utility of cfDNA assays for $\mathrm{CH}$ detection and surveillance remains poorly characterized. Using a single CLIA-certified assay, we showed that CfDNA assay performance in detecting $\mathrm{CH}$ was limited by analytical factors, which may preclude its use for $\mathrm{CH}$ detection and surveillance; and the reliability of a single commercial cfDNA assay was limited when variants were at $\mathrm{VAF}<10 \%$.

Assay sensitivity among massive parallel sequencing platforms is a key limitation for accurate detection of variants with low allele frequency in cfDNA. ${ }^{4,6}$ Invariably, all studies showing high cfDNA accuracy for tumor genotyping overcome potential sequencing errors by utilizing deep errorcorrecting sequencing techniques with original depths of $6200 x-60000 x$ to achieve error-correcting sequencing deduplication ratios of 360X-2400X and optimized protocols to mitigate sequencing errors. ${ }^{4-6,27}$ In practice, there is still no evidence of clinical utility of cfDNA assays for detection and monitoring of variants at very low allele burdens. ${ }^{2}$ Discordance among CLIA assays has also been reported for liquid biopsies when patients' paired samples were assessed in parallel in different laboratories. ${ }^{21-23}$ Similar to our results, discordance was mainly observed in variants at average VAF of $1 \%$ (and up to $3 \%$ ) whereas higher concordance is observed at VAF $>10 \% \cdot{ }^{21-23}$ In contrast to the situation in malignant diseases, unbiased $\mathrm{CH}$ detection and quantification in asymptomatic in- 
dividuals or in a disease with a low allele burden may be more challenging because cfDNA levels are typically lower in healthy individuals and are proportional to an allele burden and cell turnover. ${ }^{28,29}$ Additionally, a cfDNA yield in AA is low because of the lack of hematopoietic cells. As seen in our MDS cohort, sensitivity may not be critical when cfDNA is used for molecular profiling of malignant hematologic diseases with high allele burdens. In contrast, for asymptomatic individuals with CHIP and for AA patients, a minimal threshold of $10 \%$ for detecting de novo variants may not be useful, and reliable $\mathrm{CH}$ detection and quantification may be better achieved by screening of blood cells.

The frequency of $\mathrm{CH}$ in cfDNA from healthy individuals

A

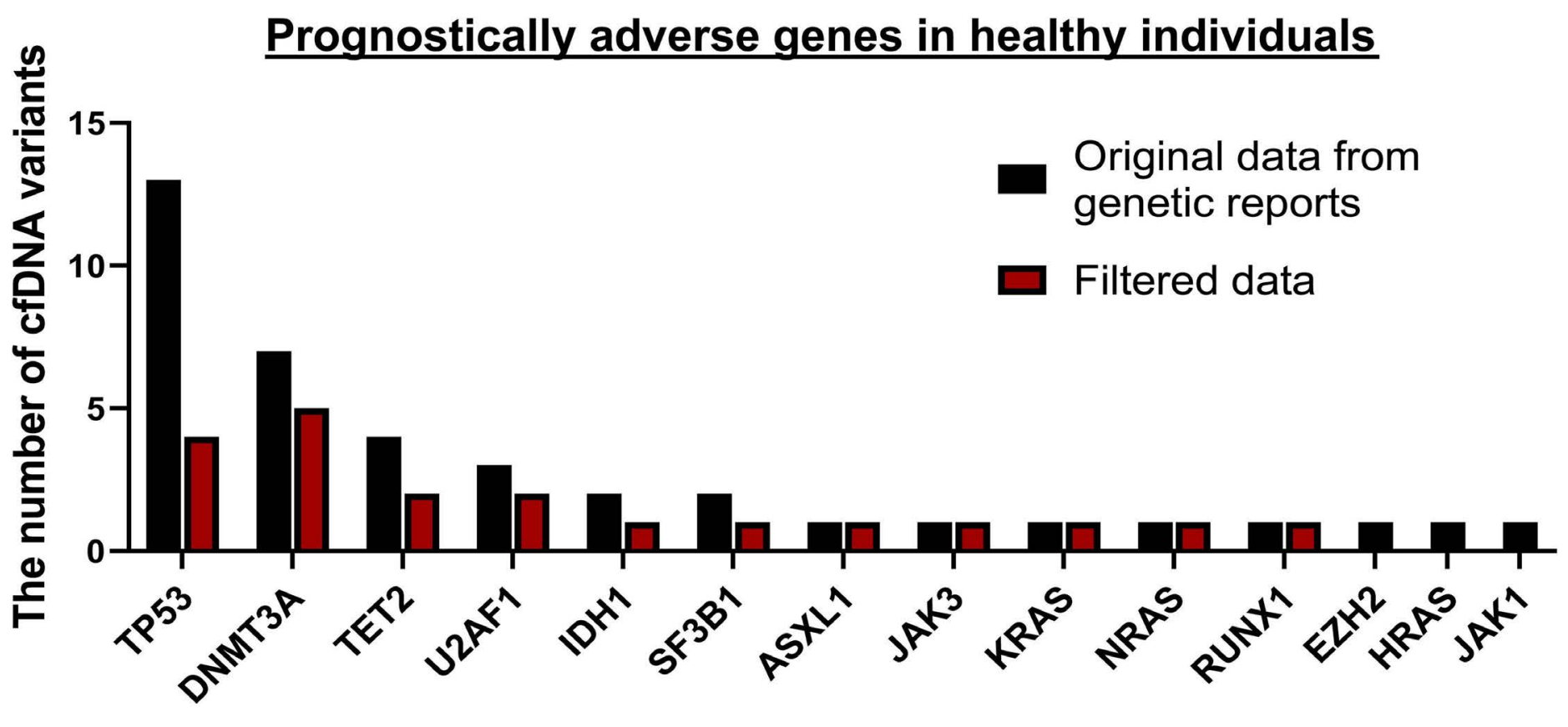

B

Retrospective clinical data in the Baltimore Longitudinal Study of Aging

\begin{tabular}{|c|c|c|c|c|c|c|}
\hline ID & $\begin{array}{l}\text { Age at } \\
\text { time of } \\
\text { sample } \\
\text { collection }\end{array}$ & $\begin{array}{l}\text { Year of } \\
\text { sample } \\
\text { collection }\end{array}$ & Clinical data & cfDNA variant & VAF & $\begin{array}{l}\text { Read } \\
\text { depth }\end{array}$ \\
\hline $\mathrm{HC}-04$ & 70 & 2018 & Prostate cancer in 2018 & IDH1: Asp390Gly & $11.7 \%$ & 60 \\
\hline $\mathrm{HC}-19$ & 61 & 2009 & $\begin{array}{l}\text { History of carcinoma in } 2003 \text { and } \\
\text { lung cancer in } 2005 .\end{array}$ & RUNX1: Arg162Gly & $1.46 \%$ & 412 \\
\hline $\mathrm{HC}-26$ & 83 & 2010 & $\begin{array}{l}\text { History of thrombocytopenia in } \\
2007 \text { and liver cysts in 2008. Died } \\
\text { of acute myeloid leukemia in } 2012 .\end{array}$ & $\begin{array}{l}\text { KRAS: Gly12Asp/ } \\
\text { UA2F1: Ser34Phe }\end{array}$ & $\begin{array}{l}31 \% / \\
51 \%\end{array}$ & $\begin{array}{l}450 / \\
333\end{array}$ \\
\hline $\mathrm{HC}-47$ & 77 & 2014 & $\begin{array}{l}\text { No history of myeloid malignancy } \\
\text { in a recent follow-up }\end{array}$ & TP53: Cys238Arg & $1.24 \%$ & 564 \\
\hline $\mathrm{HC}-51$ & 37 & 2004 & $\begin{array}{l}\text { No history of myeloid malignancy } \\
\text { in a recent follow-up. }\end{array}$ & $\begin{array}{c}\text { TP53: } \\
\text { Lys382AsnfsTer40 }\end{array}$ & $1.14 \%$ & 438 \\
\hline HC-64 & 74 & 2006 & $\begin{array}{l}\text { History of anemia since } 2006 \text { and } \\
\text { thrombocytopenia since } 2015\end{array}$ & TP53: Thr123Ala & $50 \%$ & 202 \\
\hline $\mathrm{HC}-86$ & 55 & 2006 & $\begin{array}{l}\text { Underwent chemotherapy in } 1998 \\
\text { and has a history of anemia since } \\
1996 .\end{array}$ & TP53: His296Arg & $1.22 \%$ & 411 \\
\hline $\mathrm{HC}-104$ & 76 & 2019 & $\begin{array}{l}\text { No history of myeloid malignancy } \\
\text { in a recent follow-up. }\end{array}$ & UA2F1: Lys15Glu & $17 \%$ & 65 \\
\hline
\end{tabular}

Figure 4. Healthy individuals with cell-free DNA variants in prognostically adverse genes. (A) The number of cell-free DNA (cfDNA) variants in prognostically adverse genes that were reported in the original genetic reports (black columns) and filtered by stringent criteria (red columns). (B) Retrospective clinical data from individuals enrolled in the Baltimore Longitudinal Study of Aging who were found to have variants in clonal hematopoiesis of indeterminate potential (CHIP) genes associated with an increased risk of myeloid malignancies. VAF: variant allele frequency. 
was higher than that in blood cells reported in previous studies $(60 \%$ of individuals $>40$ years old vs. $10 \%$ of individuals $>60$ years old). ${ }^{9,12}$ However, this discrepancy may be explained by analytical factors related to the cfDNA screening, such as the use of a high sensitivity DNA sequencing approach and the increased number of false positive variants found at very low VAF in CfDNA. After we filtered the data to remove likely discordant variants, the frequency of $\mathrm{CH}$ in cfDNA from healthy individuals $>40$ years old was $30 \%$, which was consistent with the data from error-correcting sequencing studies. ${ }^{14,16,24}$ Interestingly, after data filtering healthy individuals were predominantly found with single CHIP variants, as opposed to the initial data showing they were more frequently observed with two or more variants.

Since we classified cfDNA variants as false positives solely based on their presence in matched blood cells and the cfDNA pool including circulating DNA released from other tissues, it is possible that variants labeled as false positive may be truly present but not be of a hematopoietic origin. Thus, an accurate and precise assay as well as characterization of the clonal cfDNA landscape in healthy people is critical for interpretation of results. In individuals from BLSA, who had long-term follow-up and from whom a comprehensive clinical history was available, cfDNA variants likely to be related to a malignant disease were not found in the most commonly mutated CHIP genes. True positive variants at VAF $<10 \%$ were mainly observed in $A A$ (Figure 2B, C), but increased concordance in this specific setting may be explained by the fact that their cfDNA and BM samples were the only ones analyzed and reported in parallel by the laboratory. The knowledge of parallel BM results or previous data may have influenced cfDNA results as opposed to a true de novo detection (independently sequenced, analyzed and reported).

Accurate $\mathrm{CH}$ quantification in cfDNA is also critical for CHIP surveillance and therapeutic decisions in both AA and MDS. Although cfDNA often correlates with blood cells for quantification of clones, ${ }^{4,6,30}$ agreement by BlandAltman analysis showed poor agreement in quantification of mutant allele burdens between cfDNA and blood cells, suggesting that these DNA sources were not interchangeable for monitoring clonal dynamics. These findings could mislead correct interpretation of patients' clonal dynamics as the clone size is an important genomic marker of progression to myeloid malignancies.

We could not assess association of the cfDNA profile with clinical outcomes and a cardiovascular risk, due to small sample sizes of the cohorts. Lack of paired samples from healthy individuals and other clinical samples for an in-house deep error-correcting sequencing validation was the major limitation of this study. Here, the hematopoietic origins of cfDNA variants found in BLSA individuals were only validated in PBMC. AL- though previous studies have shown that either PB and $B M$ cells can be used for the detection and quantification of $\mathrm{CH}^{10,31,32}$ whether PBMC and granulocytes mirror BM as matched samples is still uncertain. We did not investigate whether biological differences accounted for cfDNA discordance with blood cells, nor did we assess the contribution of copy number variations for $\mathrm{CH}$ as cfDNA was not suitable for its detection. Of note, the commercial cfDNA assay used in this study was only validated for screening of myeloid malignancies and AA, not of healthy individuals. Nevertheless, lack of concordance was also seen in AA and MDS, suggesting that biological factors were not the main explanation of the assay discordance.

To our knowledge, this is the first study that assessed the performance of a commercial cfDNA assay for the detection and quantification of $\mathrm{CH}$ in $\mathrm{AA}$ and MDS patients in parallel with healthy individuals. Although cfDNA tests have emerged as a non-invasive alterative to BM biopsies, our data suggest that their performance was not superior to the use of BM cells for $\mathrm{CH}$ profiling of diseases marked by variants present at low allele burdens. However, our data were derived from the use of a single commercial cfDNA assay, and its performance may not be comparable to that of other commercial assays. Nevertheless, the clinical use of cfDNA for detection and surveillance of $\mathrm{CH}$ in healthy individuals showed no advantage over the use of PB cells (additionally, both of these methods require the same PB sampling). The use of a cfDNA assay for de novo $\mathrm{CH}$ detection may be limited to diseases with a high allele burden (such as MDS) or retrospective studies for which plasma is the only source of DNA. Still, clinical reports must be interpreted with caution, especially when reporting de novo cfDNA variants at low levels; up to $50 \%$ of variants from the cfDNA reports of low VAF were likely artifacts. Ultra-sensitive assays with robust sequencing coverage and error-correction methodology may be required to overcome assay discordance; however, the commercial implementation of such assays may still be prohibited by costs. Although the primary focus of this study was not the screening of circulating tumor DNA, insights into methodology illustrated in our work could be applied to solid tumors when the circulating tumor DNA burden in plasma is low and detection of very small clones is required.

\section{Disclosures}

No conflicts of interest to disclose.

\section{Contributions}

$F G R, I B, L F, S K$ and NSY made substantial contributions to the conception or design of the work; FGR, IB, DQR, EMG, $B A P$ and NS contributed to the acquisition, analysis, or interpretation of data; $L W D$ and CSH contributed to the vali- 
dation of commercial results; FGR, IB, EMG, BAP and NSY drafted the work or revised it critically for important intellectual content; FGR, IB, LF and NSY contributed to the final approval of the version to be published.

\section{Acknowledgments}

The authors would like to thank the DNA Sequencing and Genomics Core Facility at NHLBI, and Toshiko Tanaka and Linda Zukley at the NIA for their assistance.

\section{Funding}

This work was funded by the Intramural Research Program of the NHLBI/NIH and by the Intramural Research Program of the National Institute on Aging, NIH Baltimore. Research funding was provided by Novartis by way of a Cooperative Research and Development Agreement (CRADA).

\section{Data-sharing}

The human sequence data generated in this study are not publicly available due to patient privacy requirements but are available from the corresponding author upon reasonable request. Other data generated in this study are available within the article and its Online Supplementary Data Files.

\section{References}

1. Wan JCM, Massie C, Garcia-Corbacho J, et al. Liquid biopsies come of age: towards implementation of circulating tumour DNA. Nat Rev Cancer. 2017;17(4):223-238.

2. Merker JD, Oxnard GR, Compton C, et al. Circulating tumor DNA analysis in patients with cancer: American Society of Clinical Oncology and College of American Pathologists joint review. J Clin Oncol. 2018;36(16):1631-1641.

3. Condoluci A, Rossi D. The future of cell-free DNA testing to guide therapeutic decisions in B-cell lymphomas. Curr Opin Hematol. 2019;26(4):281-287.

4. Razavi P, Li BT, Brown DN, et al. High-intensity sequencing reveals the sources of plasma circulating cell-free DNA variants. Nat Med. 2019;25(12):1928-1937.

5. Li BT, Janku F, Jung B, et al. Ultra-deep next-generation sequencing of plasma cell-free DNA in patients with advanced lung cancers: results from the Actionable Genome Consortium. Ann Oncol. 2019;30(4):597-603.

6. Liu J, Chen X, Wang J, et al. Biological background of the genomic variations of cf-DNA in healthy individuals. Ann Oncol. 2019;30(3):464-470.

7. Jaiswal S, Fontanillas P, Flannick J, et al. Age-related clonal hematopoiesis associated with adverse outcomes. $N$ Engl $J$ Med. 2014;371(26):2488-2498.

8. Young AL, Challen GA, Birmann BM, Druley TE. Clonal haematopoiesis harbouring AML-associated mutations is ubiquitous in healthy adults. Nat Commun. 2016;7:12484.

9. Jaiswal S, Libby P. Clonal haematopoiesis: connecting ageing and inflammation in cardiovascular disease. Nat Rev Cardiol. 2020;17(12):828.

10. Miller PG, Steensma DP. Implications of clonal hematopoiesis for precision oncology. JCO Precis Oncol. 2020;6(4):639-646.

11. Steensma DP. The clinical challenge of idiopathic cytopenias of undetermined significance (ICUS) and clonal cytopenias of undetermined significance (CCUS). Curr Hematol Malig Rep. 2019;14(6):536-542.

12. Genovese G, Kähler AK, Handsaker RE, et al. Clonal hematopoiesis and blood-cancer risk inferred from blood DNA sequence. N Engl J Med. 2014;371(26):2477-2487.

13. Jaiswal S, Natarajan P, Silver AJ, et al. Clonal hematopoiesis and risk of atherosclerotic cardiovascular disease. N Engl J Med. 2017;377(2):111-121.

14. Abelson S, Collord G, Ng SWK, et al. Prediction of acute myeloid leukaemia risk in healthy individuals. Nature.

2018;559(7714):400-404.

15. Desai P, Mencia-Trinchant N, Savenkov O, et al. Somatic mutations precede acute myeloid leukemia years before diagnosis. Nat Med. 2018;24(7):1015-1023.

16. Jaiswal S, Ebert BL. Clonal hematopoiesis in human aging and disease. Science. 2019;366(6465):eaan4673.

17. Bejar R. Clinical and genetic predictors of prognosis in myelodysplastic syndromes. Haematologica. 2014;99(6):956-964.

18. Bejar R, Stevenson KE, Caughey B, et al. Somatic mutations predict poor outcome in patients with myelodysplastic syndrome after hematopoietic stem-cell transplantation. J Clin Oncol. 2014;32(25):2691-2698.

19. Yoshizato T, Dumitriu B, Hosokawa K, et al. Somatic mutations and clonal hematopoiesis in aplastic anemia. N Engl $J$ Med. 2015;373(1):35-47.

20. Steensma DP. How I use molecular genetic tests to evaluate patients who have or may have myelodysplastic syndromes. Blood. 2018;132(16):1657-1663.

21. Stetson D, Ahmed A, Xu X, et al. Orthogonal comparison of four plasma NGS tests with tumor suggests technical factors are a major source of assay discordance. JCO Precis Oncol. 2019;3(3):1-9.

22. Torga G, Pienta KJ. Patient-paired sample congruence between 2 commercial liquid biopsy tests. JAMA Oncol. 2018;4(6):868-870.

23. Kuderer NM, Burton KA, Blau S, et al. Comparison of 2 commercially available next-generation sequencing platforms in oncology. JAMA Oncol. 2017;3(7):996-998.

24. Jaiswal S, Libby P. Clonal haematopoiesis: connecting ageing and inflammation in cardiovascular disease. Nat Rev Cardiol. 2020;17(3):137-144.

25. Bland JM, Altman DG. Statistical methods for assessing agreement between two methods of clinical measurement. Lancet. 1986;1(8476):307-310.

26. Hourigan CS, Dillon LW, Gui G, et al. Impact of conditioning intensity of allogeneic transplantation for acute myeloid leukemia with genomic evidence of residual disease. J Clin Oncol. 2020;38(12):1273-1283.

27. Rossi D, Diop F, Spaccarotella E, et al. Diffuse large B-cell lymphoma genotyping on the liquid biopsy. Blood. 2017;129(14):1947-1957.

28. Alborelli I, Generali D, Jermann P, et al. Cell-free DNA analysis in healthy individuals by next-generation sequencing: a proof of concept and technical validation study. Cell Death Dis. 2019;10(7):534.

29. Schwarzenbach H, Hoon DS, Pantel K. Cell-free nucleic acids as 
biomarkers in cancer patients. Nat Rev Cancer. 2011;11(6):426-437.

30. Albitar A, Townsley D, Ma W, et al. Prevalence of somatic mutations in patients with aplastic anemia using peripheral blood cfDNA as compared with BM. Leukemia. 2018;32(1):227-229.

31. Guermouche H, Ravalet N, Gallay N, et al. High prevalence of clonal hematopoiesis in the blood and bone marrow of healthy volunteers. Blood Adv. 2020;4(15):3550-3557.

32. Mohamedali AM, Gäken J, Ahmed M, et al. High concordance of genomic and cytogenetic aberrations between peripheral blood and bone marrow in myelodysplastic syndrome (MDS). Leukemia. 2015;29(9):1928-1938. 